\title{
МЕТОДИЧЕСКИЙ ПОДХОД К ПРОГНОЗИРОВАНИЮ НЕРАВНОМЕРНОГО ВРЕМЕННОГО РЯДА
}

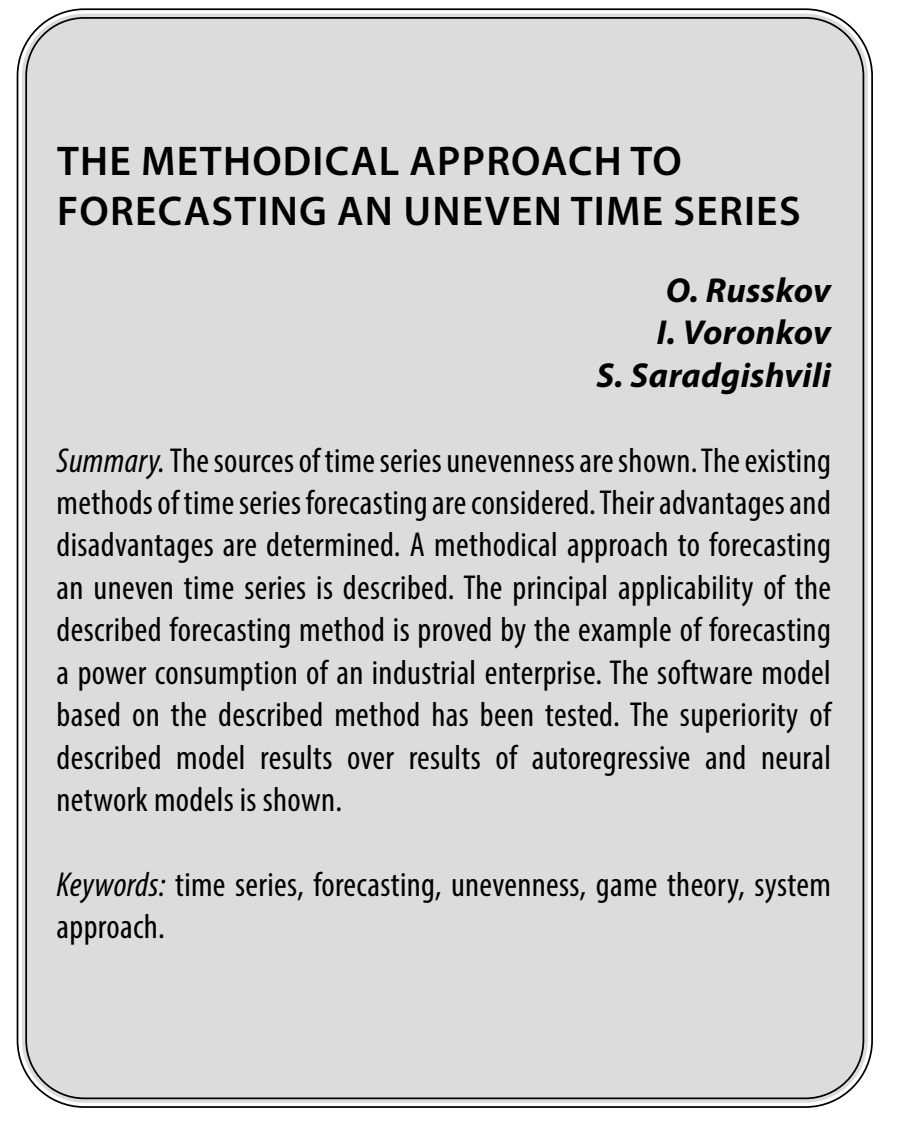

\section{Введение}

B настоящее время в мире имеется очевидный тренд на цифровизацию, в связи с чем бурное развитие переживают информационные технологии. Количество процессов, цифровые расчёты алгоритмов функционирования которых становится возможным производить в реальном времени, неумолимо растёт. В связи с этим активно развиваются и методы информационных технологий, позволяющие прогнозировать будущее состояние исследуемых объектов на базе их цифровых двойников и прогнозных моделей. Предвосхищать будущее с помощью вычислительной машины с точки зрения науки означает эффективное удовлетворение одной из базовых потребностей человека в безопасности. Поэтому не следует сомневаться в том, что развитие цифровых методов прогнозирования с использованием быстродействующих ЭВМ продолжится. При этом информация о состоянии исследуемых объектов в методах информационных технологий обычно представляется в виде
Руссков Олег Владимирович

Аспирант, Санкт-Петербургский политехнический университет Петра Великого vtz_energo@mail.ru

Воронков Илья Александрович

Аспирант, Санкт-Петербургский политехнический университет Петра Великого iliaftk@outlook.com

Сараджишвили Сергей Эрикович

К.т.н., дочент, Санкт-Петербургский политехнический университет Петра Великого SSaradg@yandex.ru

Аннотация. Показаны источники происхождения неравномерности временного ряда. Рассмотрены существующие методы прогнозирования временных рядов. Определены их достоинства и недостатки. Описан методический подход к прогнозированию неравномерного временного ряда. Обоснована принципиальная применимость описываемого метода прогнозирования на примере прогнозирования электропотребления крупного промышленного предприятия. Апробирована программная модель, созданная на основе описываемого метода. Показано превосходство результатов прогнозирования описанной модели над результатами авторегрессионной и нейросетевой моделей прогнозирования.

Ключевые слова: временной ряд, прогнозирование, неравномерность, теория игр, системный подход.

временных рядов данных, отражающих изменение определённого параметра объекта исследования во времени с заданной дискретностью. Количество данных при этом имеет тенденцию к значительному росту.

\section{$\triangle$ ОстоИнства И неАОстатКИ сушествуюших цифровых мето $о в$ прогнозирования временных ряАОв}

На данный момент для решения задачи прогнозирования временных рядов во всё мире хорошо зарекомендовали себя авторегрессионные модели ARIMA (ARIMAX) [1], модели на нейронных сетях ANN [2], модели экспоненциального сглаживания [3], на цепях Маркова [4], на базе классификационно-регрессивных деревьев CART [5]. К их достоинствам традиционно относят низкую погрешность прогнозирования, гибкость и широкую применимость. Особо следует выделить значительный рост числа исследователей, адаптирующих к различным отраслям науки и техники 


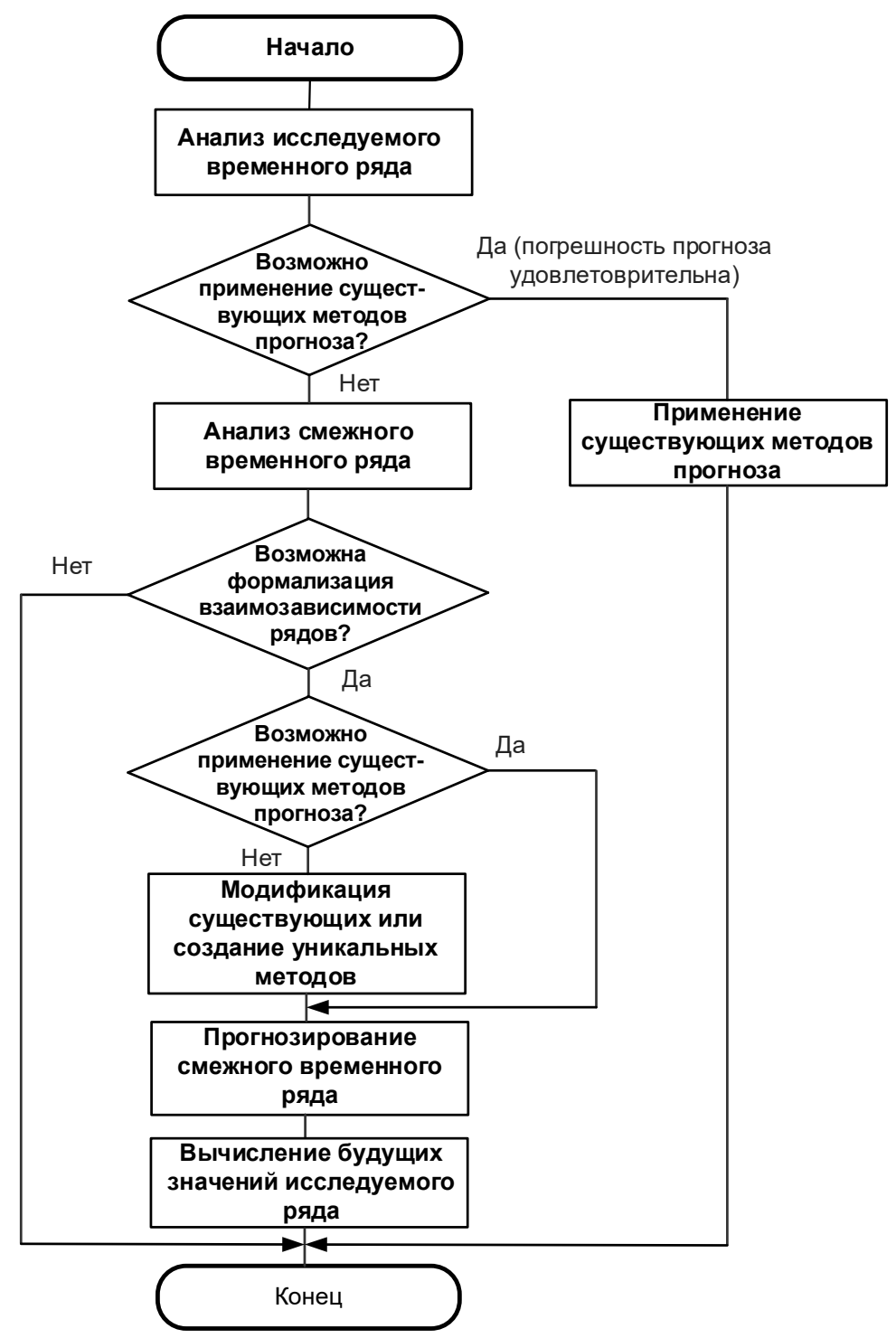

Рис. 1. Обобщённый алгоритм прогнозирования неравномерного временного ряда

нейросетевые модели. Общее научное мнение сейчас таково, что именно за этими моделями будущее. Считается, что преградой к полной цифровизации любого процесса является временное отсутствие алгоритмов оптимизации и соответствующих им вычислительных мощностей; со временем этот недостаток будет обязательно устранён. Однако, к известным недостаткам существующих моделей, в том числе нейросетевых, относится критичный рост погрешности прогноза при росте неопределённости (неравномерности [6], волатильности [7]) исследуемого ряда. И хотя адепты нейросетей утверждают, что и здесь вопрос только в правильном анализе и учёте в построении модели влияющих на процесс факторов, на данный момент озвученная проблема существующими моделями решается не всегда. На практике существует достаточное ко- личество временных рядов, имеющих неравномерную природу [8]. Это, в частности, временные ряды, связанные с производством, где основную роль играют технологии и технологи. Например, графики потребления энергоресурсов и вспомогательных материалов, график ремонтов оборудования, распределения плавок стали по часам суток и другие имеют ярко выраженную неравномерность. Анализ факторов, влияющих на возникновение подобной неравномерности, показывает решающую роль человеческого фактора [8]. Попытки его систематизировать созданием специальных моделей $[9,10]$ пока не принесли успеха. Поскольку число таких неравномерных временных рядов достаточно велико [8], создание методов и моделей их прогнозирования имеет важное научное и народнохозяйственное значение. 


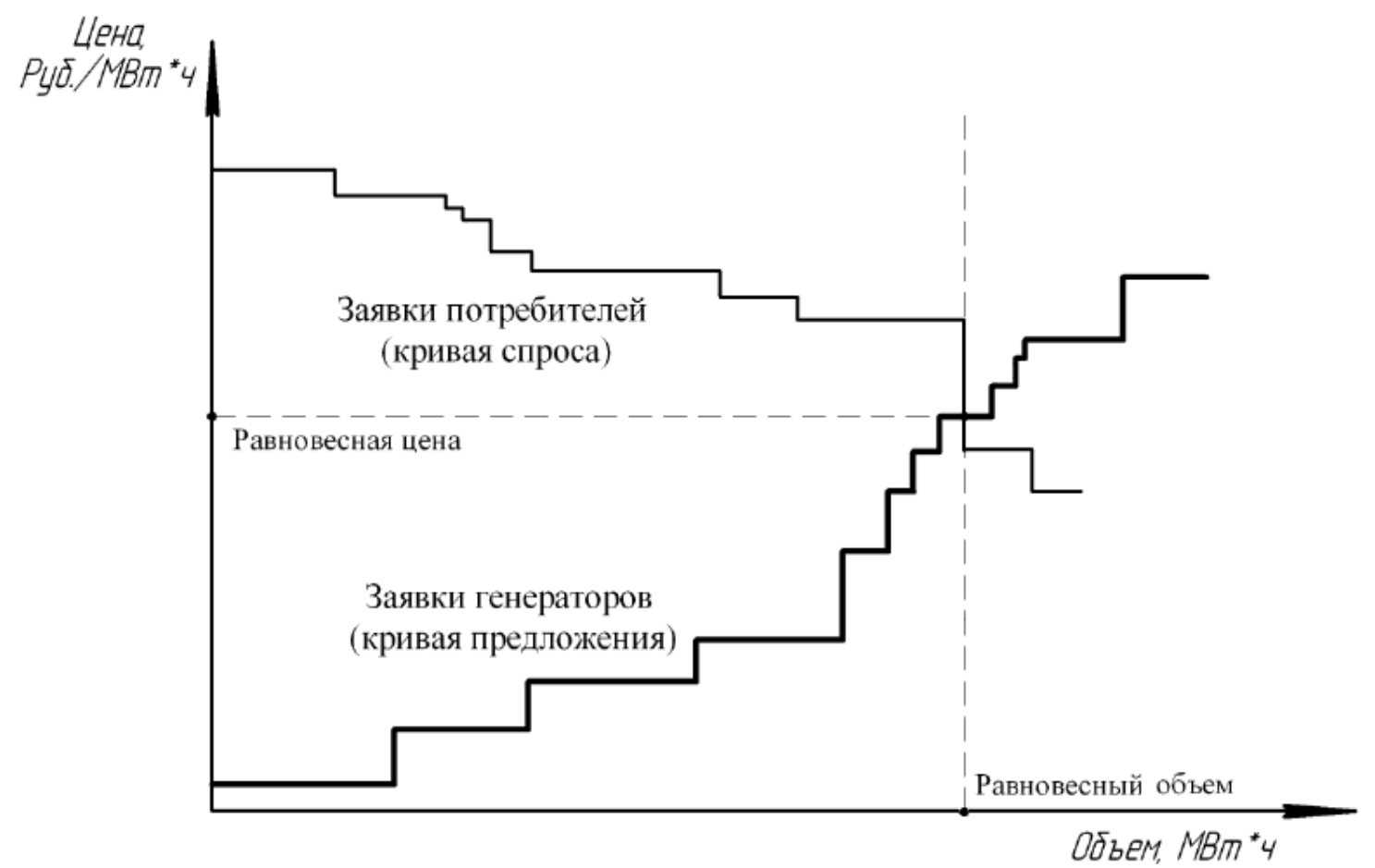

Рис. 2. Взаимосвязь временных рядов цен и электропотребления [13]

\section{МетоАический поАхо} К прогнозированию

неравномерного временного ряла

Для того, чтобы осуществить прогнозирование неравномерного временного ряда, формализация влияющих факторов которого в настоящее время слишком сложна из-за отсутствия адекватных алгоритмов их учёта, возможно применение системного подхода (рис. 1).

При этом, безусловно, должны анализироваться законы формирования и степень влияния рядов смежных областей на исследуемый ряд. Для этого требуется обработка больших массивов данных и выявление взаимосвязей [11]. В частности, могут применяться методы и платформы для совместного анализа [12]. В случае, если искомое влияние обнаруживается и может быть формализовано, анализируется характер смежного ряда. Если смежный ряд более равномерный, чем исследуемый, и его значения можно спрогнозировать с требуемой погрешностью, то осуществляется прогноз значений смежного ряда, после чего с помощью формализованной зависимости происходит вычисление искомых значений исследуемого ряда. При этом область смежного процесса может отличаться от области, в которой формируется основной процесс. Самым ярким примером могут служить графики цен на энергоносители и объёмы их выработки или потребления. Первый процесс относится к области экономики, вто- рой - энергетики. Процесс прогнозирования подобных неравномерных рядов укладывается в рамки описываемой методологии.

\section{Применение метоло^огии}

В качестве примера применения вышеописанной методологии рассмотрим наиболее часто встречающуюся связку рядов - электропотребления промышленного предприятия и цен на электрическую энергию на оптовом рынке (рис. 2).

Ряд часового электропотребления обладает ярко выраженной неравномерностью вследствие превалирования требований технологии производства над требованиями часового планирования на оптовом рынке электроэнергии; вследствие этого его прогнозирование с помощью существующих моделей имеет значительную (10-15\%) погрешность [8]. Формализация влияющих факторов затруднена вследствие превалирования в их структуре человеческого фактора. Согласно рассматриваемой методологии следует рассмотреть смежные ряды, имеющую сходную дискретность. Из области экономики можно выделить перспективные в этом отношении временные ряды цен на рынке на сутки вперёд и на балансирующем рынке [14]. Их дискретность совпадает с дискретностью исследуемого ряда; при этом общность природы формирования рядов гарантирует механизм электронного аукциона [14]. 


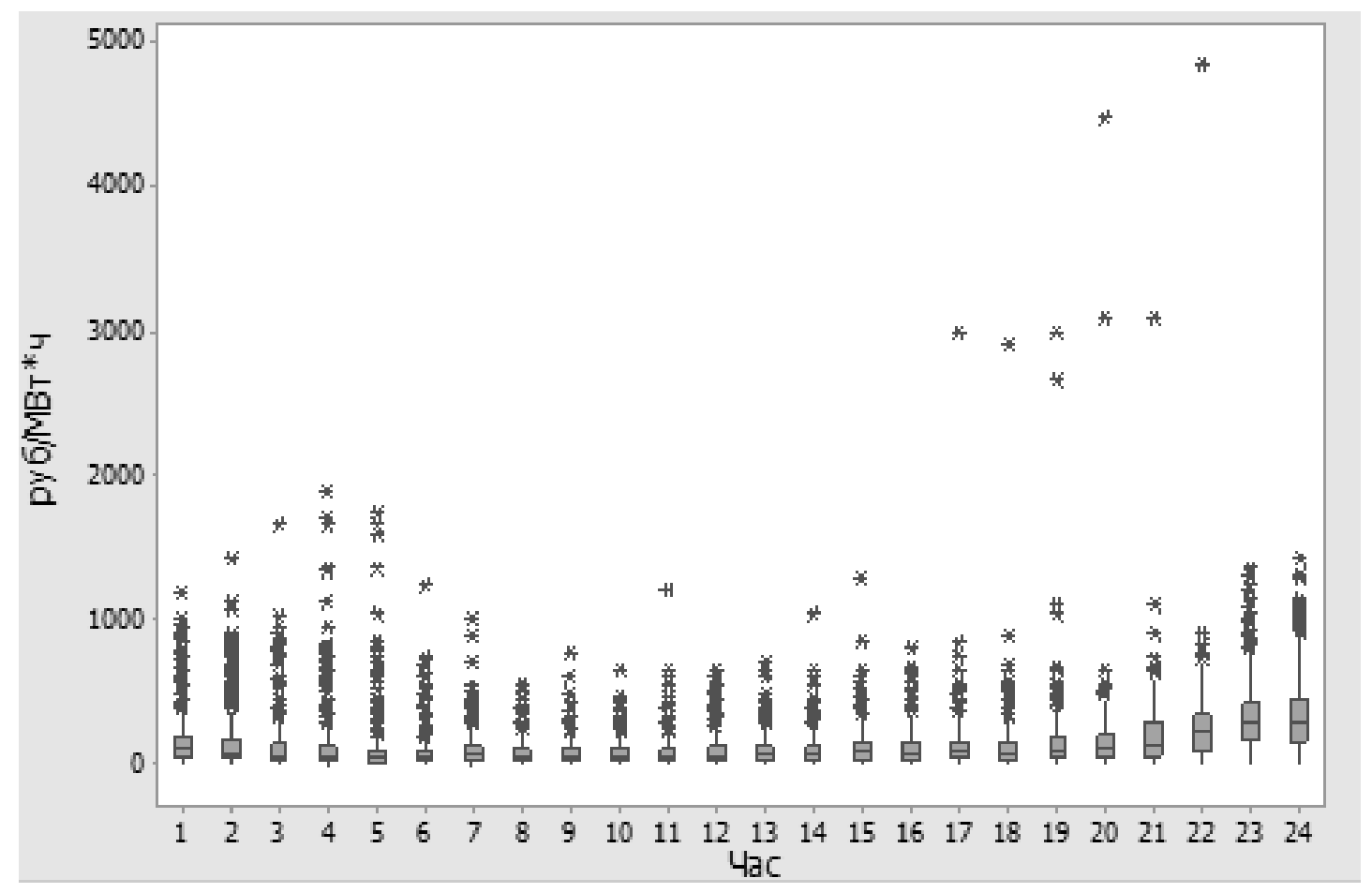

Рис. 3. Изменчивость значений смежных временных рядов

Для формализации зависимости этих рядов были применены теория принятия решений и теория игр, которые позволили выявить и оцифровать связь с помощью формулы полной вероятности, описывающей все возможные исходы детерминированного эксперимента по фиксации всех возможных взаимных отношений значений временных рядов часового электропотребления и цен рынка. Детальный вывод формулы был описан авторами ранее [15]:

$$
\begin{aligned}
& F_{0}=V \cdot\left(P_{1} \cdot \Delta_{0}-P_{2} \cdot \Delta_{1}+P_{1} \cdot P_{2} \cdot\left(\Delta_{1}-\Delta_{0}\right)-\right. \\
& \left.-2 \cdot P_{2} \cdot C_{\kappa o p}+C_{\kappa o p}\right)
\end{aligned}
$$

где $P_{1}$ - вероятность формирования соотношения значений смежного временного ряда; $P_{2}$ - вероятность соотношения значений исследуемого временного ряда; $V$ - величина корректировки значений исследуемого ряда; $\Delta_{0}, \Delta_{1}$ - средняя разница значений смежного ряда для соотношений типов 0 и 1 соответственно; $C_{\text {кор }}$ - дополнительное корректирующее значение смежного временного ряда [15].

Знак и объём корректировки прогнозного значения неравномерного ряда определяются на основе значений целевой функции, описывающей зависимость исследуемого и смежного рядов (1). Прогнозирование происходит отдельно по каждому часу суток. Поскольку статистический анализ разницы значений смежных рядов цен показывает разную изменчивость в зави- симости от часа суток, такой подход правомерен, поскольку учитывает данную изменчивость (рис. 3).

Следование выведенной зависимости обеспечивает обоснованное в соответствии со свойством статистической устойчивости преобладание прибыли над убытком целевой функции процесса прогнозировании временного ряда неравномерного электропотребления. Алгоритм процесса вычисления прогнозного значения неравномерного временного ряда электропотребления на основе значений смежного ряда цен показан на рис. 4.

АПРОБАЦИЯ МЕТОДА НА ОСНОВЕ ОПИСАННОГО ПОДХОДА. Испытания алгоритмов модели прогнозирования неравномерного временного ряда на примере прогнозирования электропотребления крупного промышленного предприятия осуществлены на промышленном предприятии в Волгоградской области. Алгоритмы реализованы в виде программного модуля на языке $\mathrm{C}++$ (4075 строк исходного кода), исполняемого на IBM РС-совместимом компьютере с операционной системой Windows XP и выше. На программный модуль в установленном порядке получено свидетельство о государственной регистрации программ для ЭВM.

Основные результаты работы предложенных моделей, выводимые на экран: 


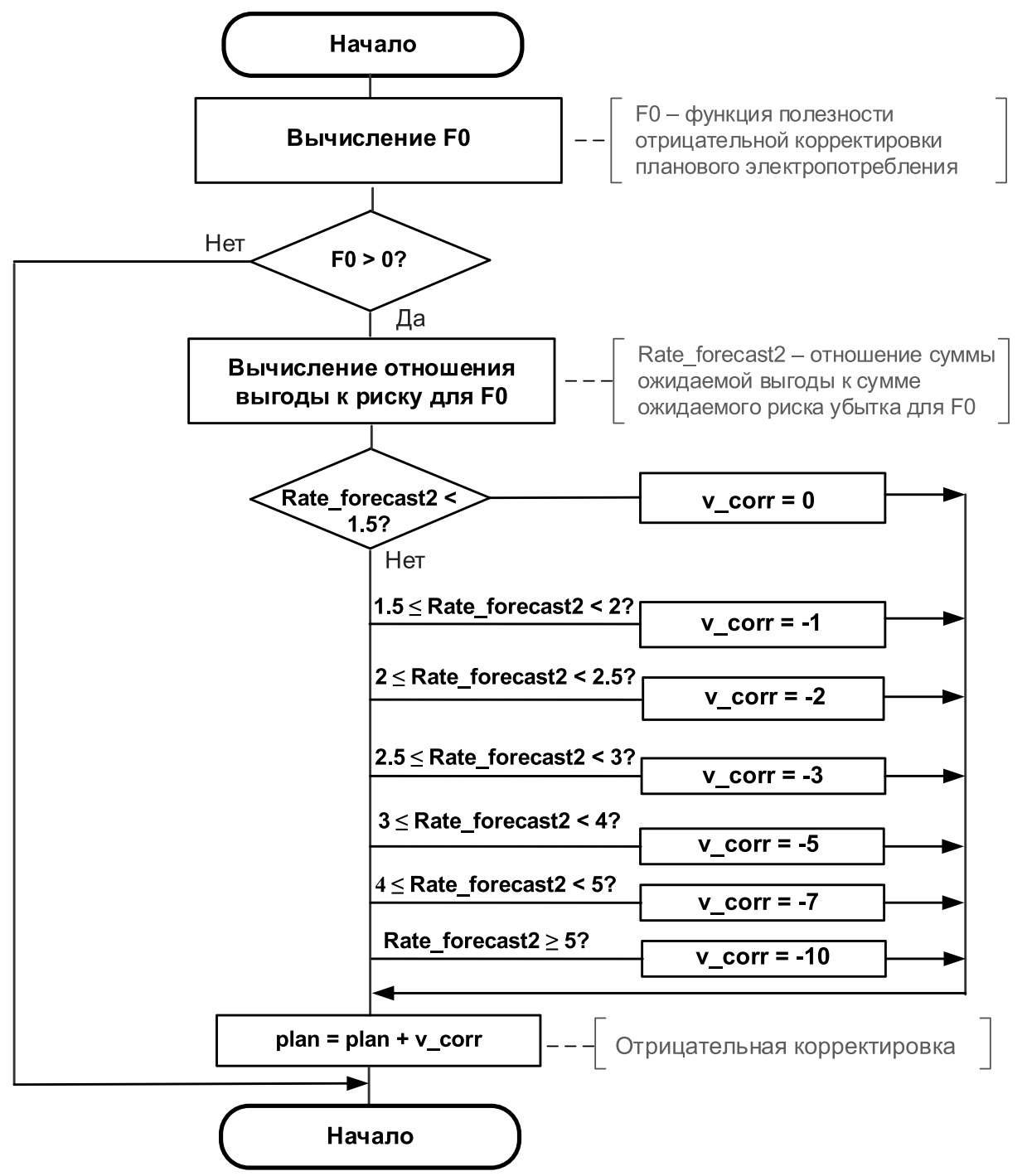

Рис. 4. Алгоритм вычисления прогнозного значения неравномерного временного ряда

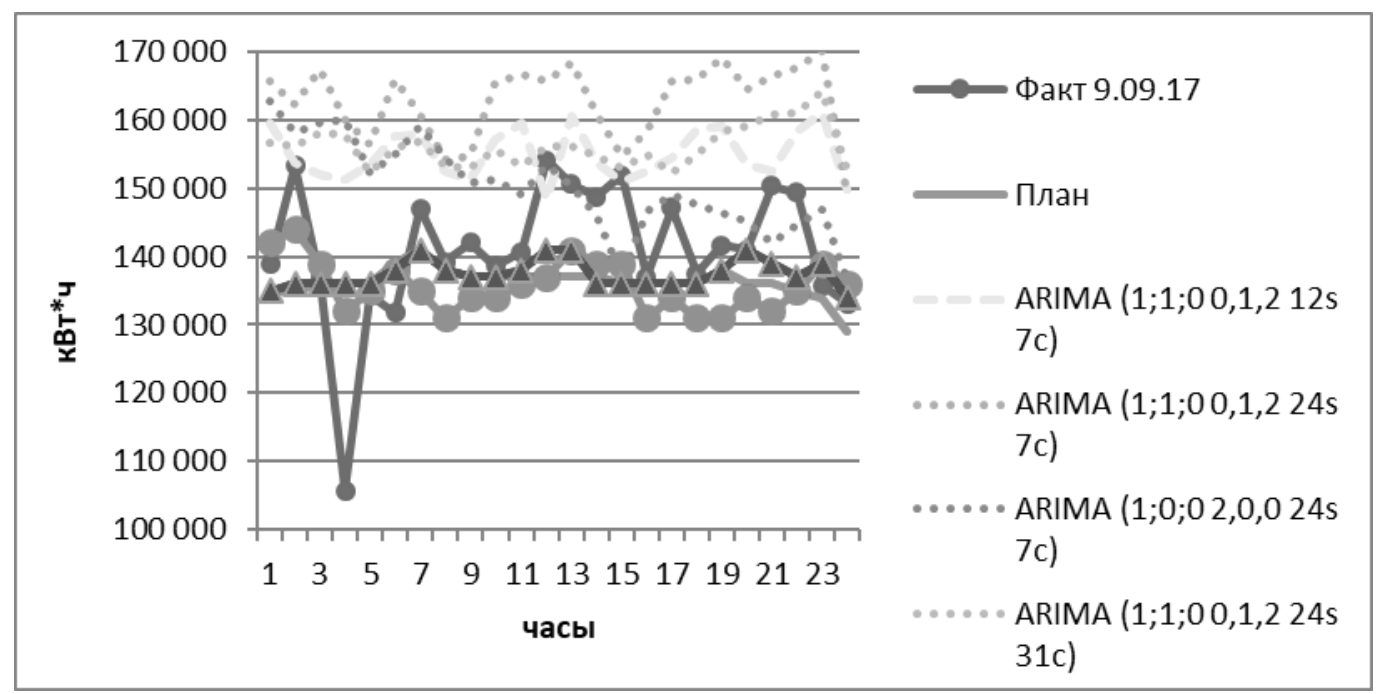

Рис. 5. Лучший суточный результат разработанной модели на основе теории игр 
- вероятность формирования прогнозируемых соотношений смежных временных рядов для лица, принимающего решение об итоговом прогнозе неравномерного временного ряда;

- итоговый результат прогнозирования неравномерного временного ряда;

- разница суммарных отклонений прогнозных и фактических значений;

- визуализация статистических значений неравномерного и смежных временных рядов;

- визуализация типов соотношений временных рядов.

Результаты работы разработанной модели прогнозирования были подвергнуты сравнению с результатами самых популярных из существующих на сегодняшний день моделей - нескольких модификаций авторегрессионной ARIMA и нейросетевой ANN. B качестве периода оценки результатов выбран 1 типовой месяц; при этом обосновано, что данные в нём являются репрезентативными для выборки целого года. По результатам испытаний лучший результат среди набора моделей показала разработанная модель на основе теории игр (рис. 5): ошибка прогноза МАE на 6,3\% меньше, чем у ANN и 33,9\% - чем у лучшей модификации $\operatorname{ARIMA}(1,1,00,1,212 \mathrm{~s} 7 \mathrm{c})$.

\section{Выво $\triangle \mathrm{b}$}

Описанный методический подход, методы и модели, создаваемые на его основе, и, в частности, описанный пример прогнозирования неравномерного электропотребления крупного промышленного предприятия, способствуют развитию цифровой энергетики EnergyNet, позволяют снижать углеводородный след в производимой в России электроэнергии за счёт разгрузки неэффективных генераторов. Кроме того, за счёт формализации связи смежных рядов промышленности описанный подход принципиально может использоваться при переходе на ритмичное бережливое производство («Цифровое производство»). Вышеизложенное позволяет считать описанный методический подход перспективным, а методы, созданные на его основе, решающими возложенные задачи по прогнозированию неравномерных временных рядов.

\section{ЛИТЕРАТУРА}

1. Berk K. Modeling and Forecasting Electricity Demand — A Risk Management Perspective. — Wiesbaden: Springer-Spektrum. — 2015.— p. 115.

2. Campillo J., Wallin F., Torstensson D., Vassileva I. Energy demand model design for forecasting electricity consumption and simulating demand response scenarios in Sweden // International Conference in Applied Energy. — 2012. — ICAE-4 ID: ICAE2012-A10599.

3. Zahan R., Kennet S.R. Modeling and Forecasting Energy Consumption in the Manufacturing Industry in South Asia // Int. Journal of Energy Economics and Policy.-3-1.-2013.—pp. 87-98.

4. Zhou X., Tang Y., Xie Y., Li Y., Zhang H. A Fuzzy Probability-based Markov Chain Model for Electric Power Demand Forecasting of Beijing, China // Energy and Power Engineering. - 2013. - 5.- pp. 488-492.

5. Кирилова Т., Бабинович Д., Климова Г., Шутов Е. Многофакторное прогнозирование потребления электроэнергии в промышленном и бытовом секторах // Пром. энергетика. - 2010. - N6. - c. 24-30.

6. Макоклюев Б.И., Ёч. В.Ф. Взаимосвязь точности прогнозирования и неравномерности графиков электропотребления // Электрические станции.2005. - № 5.- С. 64-67.

7. Бейден С., Смирнов Д., Матвеева М. Риски на рынке электроэнергии и методы прогнозирования цен // Энергорынок. — 2004. — № 4. — c. 22-28.

8. Руссков 0.В., Сараджишвили С.Э. Ограничения математических моделей в прогнозировании неравномерных временных рядов и пути их преодоления // НАУ. - 2014. — № 4. - С. 55-59.

9. Канеман Д. Думай медленно... решай быстро.— М.: АСТ, 2013.— 656 с.

10. Rabin M. Psychology and economics // Journal of economic literature. - 1998. - Vol. 36 No. 1, pp. 11-46.

11. Воронков И.А., Сараджишвили С.Э. Microsoft power BІ в цифровой обработке многомерных сигналов // Издательство СПбПУ им. Петра Великого, Санкт-Петербург, 2019, 32 стр. 一 ил.

12. Voronkov I.A., Saradgishvili S.E. Usage of a BART algorithm and cognitive services to research collaboration platforms // Journal of Physics: Conference Series, 2020, 1694(1), 012028.

13. Дзюба А.П. Управление энергозатратами промышленных предприятий: дис. ... канд. техн. наук. — Челябинск. — $2014 .-286$ c.

14. Регламенты оптового рынка электроэнергии [электронный ресурс].— https://www.np-sr.ru/ru/regulation/joining/reglaments/index.htm (дата доступа 17.02.2021)

15. Russkov 0.V., Saradgishvili S.E. The method of planning the energy consumption for electricity market // IOP Conference Series: Earth and Environmental Science 90 (2017) 012068. — 2017.—DOI: 10.1088/1755-1315/90/1/012068 